\title{
Estudio del comportamiento de una carpeta de rodado estabilizada con cloruro de calcio
}

\author{
Study of the behaviour of an unpaved road stabilized with calcium chloride
}

\section{Eduardo Morales y Carlo Pailacura}

Fecha de entrega: 1 de abril 2019

Fecha de aceptación: 2 de septiembre 2019

Departamento de Ingeniería Civil, Universidad Católica del Norte, Avda. Angamos 0610, Antofagasta, Chile, emorales@ucn.cl, carlo.pailacura@gmail.com

Un alto porcentaje de los caminos de la región de Antofagasta no se encuentran pavimentados, y según la Dirección de Vialidad más del $40 \%$ no están en buenas condiciones. Los caminos no pavimentados experimentan un deterioro más acelerado que un camino pavimentado. Esto se debe a que las partículas finas al juntarse con los agregados gruesos, expuestos al medio ambiente pierden humedad, sumado a la acción abrasiva del tránsito vehicular, generan un disgregamiento superficial del material convirtiéndose en polvo y originando fallas tales como baches, ondulaciones y calaminas, lo cual trae como consecuencia pérdida de confort, seguridad y durabilidad. El objetivo de esta investigación es evaluar en terreno el cloruro de calcio $\mathrm{CaCl}_{2}$ como estabilizador de carpetas de rodados en caminos sin pavimentar, analizando el comportamiento de un camino de prueba estabilizado con $\mathrm{CaCl}_{2}$ para conocer cómo influye su incorporación en las propiedades mecánicas del suelo. La metodología empleada es la construcción de un camino de prueba con un tramo estabilizado con $\mathrm{CaCl}_{2}$ y un tramo menor estabilizado con Bischofita. De forma paralela se realizó un estudio experimental en terreno y laboratorio para evaluar la influencia del $\mathrm{CaCl}_{2}$ utilizado para la construcción del camino. Los resultados observados en terreno y en laboratorio permiten concluir que la aplicación de $\mathrm{CaCl}_{2}$ genera un mejoramiento sustantivo en el comportamiento de las capas de rodadura, debido al efecto cementante que genera sobre la superficie, mejorando las propiedades del camino, además de ser un producto económico y de fácil aplicación.

Palabras clave: estabilización de caminos, caminos no pavimentados, cloruro de calcio, estudio experimental
Currently a large percentage the roads in the Antofagasta Region are not paved, according to the MOP Highway Direction, more than $40 \%$ are not in good condition. Unpaved roads experience a more accelerated deterioration than a paved road, since the fine particles when joined with the coarse aggregates exposed to the environment, loss moisture, added to the abrasive action of vehicular traffic, generate a superficial disintegration of the material, becoming dust and causing superficial faults such as; bumps, corrugations and corners, which results in loss of comfort, safety and durability. The objective of this investigation is to evaluate in situ calcium chloride $\mathrm{CaCl}_{2}$ as a stabilizing agent for granular layers on unpaved roads, analysing the behaviour of a test road stabilized with $\mathrm{CaCl}_{2}$, to understand how its incorporation influences the mechanical properties of the soil. The methodology used is the construction of a test path with a section stabilized with $\mathrm{CaCl}_{2}$ and a section stabilized with Bischofita. In parallel, an experimental study was carried out in the field and in the laboratory, to evaluate the influence of $\mathrm{CaCl}_{2}$ used for the construction of the road. The results observed in the field and in the laboratory allow us to conclude that the application of $\mathrm{CaCl}_{2}$ generates a substantial improvement in the behaviour of the rolling layers, due to the cementing effect it generates on the surface, improving road properties, as well as being an economical product easy to apply.

Keywords: stabilization of roads, unpaved roads, calcium chloride, experimental study

\section{Introducción}

El objetivo de este estudio es evaluar in situ el comportamiento de un camino al que se le aplica cloruro de calcio $\mathrm{CaCl}_{2}$ como agente estabilizador, comparando resultados con la Bischofita que normalmente se aplica en la zona Norte de Chile como agente estabilizador en caminos no pavimentados. 
En la región de Antofagasta existen aproximadamente 2800 kilómetros de caminos de ripio y tierra, pertenecientes a la Red Vial Básica y a la Red Vial Comunal (sin considerar caminos privados pertenecientes a las empresas mineras), de los cuales dependen comunidades que no pueden mejorar su conectividad, debido a que la construcción de caminos de alto estándar no puede ser realizada, dada la baja rentabilidad que existe en estos poblados (MOP, 2014). Estos caminos no pavimentados se deterioran rápidamente por efectos climáticos o por la acción abrasiva del tránsito, provocando: pérdida de confort y seguridad, aumento de costos de operación y gasto de mantenimiento, así como también aumento en los tiempos de viaje, que se traducen en definitiva en altos costos económicos. Debido a esto, es necesario buscar alternativas para prolongar la vida útil de la carpeta de rodado de este tipo de caminos.

En los últimos años se han desarrollado nuevas tecnologías para la construcción de caminos, algunas de éstas son: imprimaciones asfálticas reforzadas, utilización de asfalto espumado, y mejoramiento del asfalto a base de reciclado de neumáticos (e.g. Botasso y Segura, 2013). Por otro lado, se han utilizado estabilizadores de carpetas granulares mediante la adición de productos como sales, dentro de las cuales destacan el cloruro de magnesio, llamado Bischofita (e.g. Thenoux y Vera, 2002) y el cloruro de calcio $\mathrm{CaCl}_{2}$ (e.g. Monlux, 2003). Este último ha sido escogido como objeto de estudio para el análisis de su comportamiento, en carpetas de rodados granulares de caminos mineros de la región de Antofagasta, debido a su disponibilidad, características climáticas del sector y propiedades de este material.

\section{Estabilización de caminos con $\mathrm{CaCl}_{2}$}

La estabilización química del suelo es un proceso que busca mejorar las propiedades mecánicas mediante la adición de un agente estabilizador o aditivo químico como el cloruro de calcio, cloruro de magnesio, cloruro de sodio, etc. (e.g. Crespo, 1998; Cortés, 2002). El estabilizante químico a utilizar dependerá del tipo de suelo y de las propiedades que se deseen mejorar buscando siempre el beneficio económico. En general la estabilización química de los suelos utilizados como base de caminos, genera aumento de su resistencia mecánica, disminución de la permeabilidad, reducción de cambios volumétricos (asentamientos o expansiones) y disminución de la emanación de polvo de la superficie.

\section{Propiedades del $\mathrm{CaCl}_{2}$}

El cloruro de calcio $\mathrm{CaCl}_{2}$ se obtiene como un subproducto en forma de salmuera en algunos procesos industriales, aunque también se puede obtener de algunos arroyos y pozos naturales, siendo la fuente más común el obtenido en la elaboración de Carbonato de Sodio mediante procedimientos químicos. Entre las principales características del cloruro de calcio está el control de humedad y mejora en resistencia mecánica del suelo.

La propiedad higroscópica y delicuescente del cloruro de calcio le confiere la capacidad de absorber humedad del aire y disolverse en esta humedad para formar una solución líquida (Occidental Chemical Chile, 2012). En este sentido el $\mathrm{CaCl}_{2} \quad$ absorberá y retendrá humedad de la atmósfera por un tiempo prolongado, cuando ésta se encuentre por encima de ciertos valores durante parte del día (Mateos, 2007). En condiciones ambientales por sobre la curva del gráfico de la Figura 1, el $\mathrm{CaCl}_{2}$ será capaz de absorber humedad y disolverse en ésta.

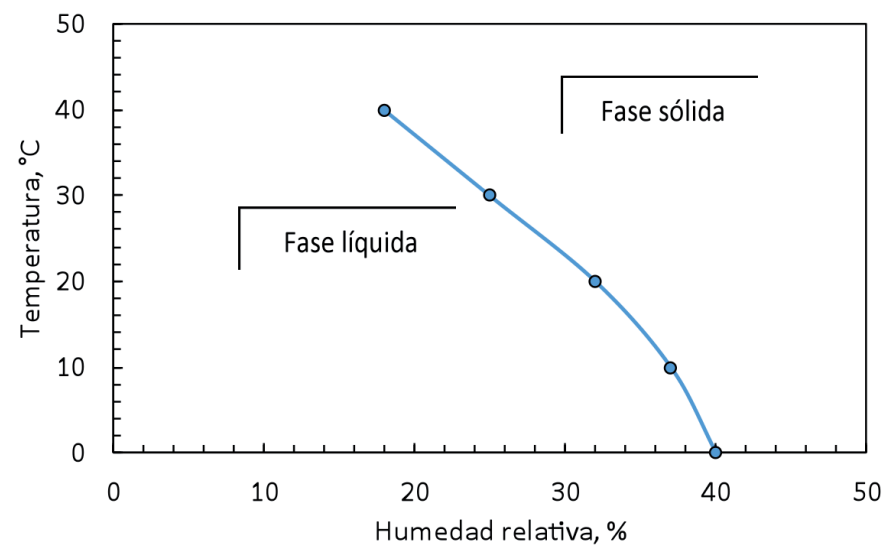

Figura 1: Condiciones ambientales para inicio de absorción de humedad del ambiente por parte del cloruro de calcio (Mateos, 2007)

Cuando la humedad relativa del ambiente se encuentra alta, por sobre los 30\%, el cloruro de calcio es capaz de absorber hasta 10 veces su propio peso (Fernández, 1982), pudiéndose mantener dicha humedad en sus dos terceras partes durante un día de calor seco.

Por otra parte, el cloruro de calcio en solución se congela a una temperatura menor que el agua pura. La temperatura de congelación de la solución depende de la concentración 
de cloruro de calcio, por cada $1 \%$ de cloruro de calcio adicionado al agua, se reduce su punto de congelación en $6^{\circ} \mathrm{C}$. Por consiguiente, los suelos tratados con cloruro cálcico sufren menos los efectos del hielo y deshielo (Mateos, 2007; Fernández, 1982).

\section{Efectos del $\mathrm{CaCl}_{2}$ en los suelos}

La estabilización con cloruro de calcio produce una cementación en la capa de rodadura con un aspecto superficial parecido a un pavimento, pero en estado de saturación el cloruro de calcio pierde su efecto cementante, el cual se recupera nuevamente cuando el material se seca (Orobio et al., 2007). A esta densificación por la acción del cloruro se le identifica como el curado de la mezcla cloruro/ suelo, porque tiene lugar después de la compactación. El aumento en densidad durante el curado está relacionado con la disminución en el espesor de las películas de agua que rodean las partículas del suelo, debido a la pérdida de parte de la humedad de la capa estabilizada. La pérdida de humedad durante el curado favorece la concentración de la solución de cloruro, aumentando la tensión superficial en la superficie, lo que contribuye al incremento en la densidad observada (Mateos, 2007).

La aplicación del cloruro de calcio en el suelo mejora la compactación del material, debido a que existe un incremento en la densidad seca máxima y una disminución en el contenido óptimo de humedad. Esto se debe al incremento de cristales del aditivo que se unen a los minerales del suelo, ayudando en la absorción de agua que antes solo lo hacía el suelo (Quinche, 2006). Por la propiedad del cloruro de calcio de absorber humedad del ambiente bajo condiciones de humedad relativa altas (durante la noche y en las mañanas), esta humedad es retenida por el suelo del camino no pavimentado, durante un periodo de tiempo dependiendo de las condiciones del ambiente, evitando la pérdida de partículas finas en forma de polvo con el paso de los vehículos y la posterior pérdida de partículas más gruesas.

Una solución de cloruro tiene una presión de vapor más baja que el agua pura, por lo que las moléculas de agua en la solución se evaporan más lentamente que las moléculas del agua pura. Debido a estas propiedades es por lo que se usa el cloruro como paliativo del polvo. La humedad que imparte la adición de cloruro a la superficie de un camino de tierra, mantiene los áridos más estables que en uno sin tratar. Al perderse menos áridos se realiza una economía importante en la conservación de los caminos (Mateos, 2007).

Para obtener el máximo beneficio del tratamiento con cloruro cálcico se requieren pocas cantidades de este producto aplicado en estado sólido o en solución (Figura 2). La cantidad de cloruro a añadir es generalmente entre 3 y $10 \mathrm{~kg}$ por tonelada métrica de suelo a tratar para la construcción de cimientos (bases y sub-bases) de pavimentos. En aplicaciones superficiales se puede emplear $0.5 \mathrm{~kg} / \mathrm{m}^{2}$, repitiendo la aplicación cada vez que se observe excesiva sequedad en la superficie de la carretera.
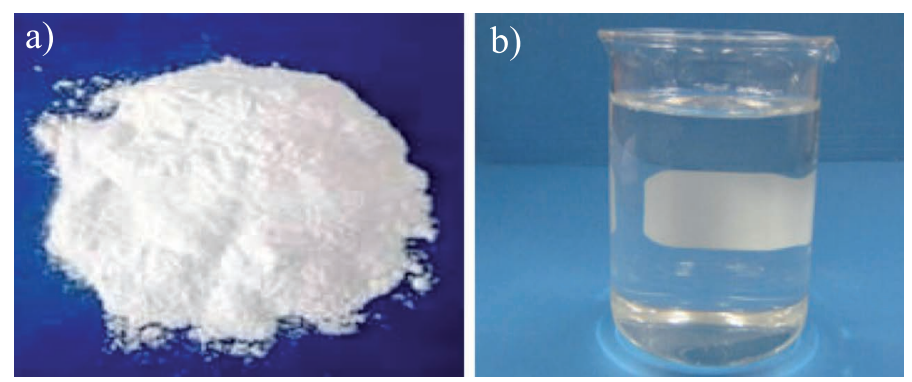

Figura 2: Cloruro de calcio en sus diferentes estados: a) polvo y b) líquido

\section{Metodología de trabajo}

La investigación tiene dos etapas: laboratorio y terreno. En laboratorio se realizaron ensayes a muestras de suelo tales como Proctor modificado, CBR, triaxial y absorción de humedad para determinar la incidencia del $\mathrm{CaCl}_{2}$ en las propiedades mecánicas del suelo del tramo de camino en estudio. La investigación en terreno consistió en evaluar periódicamente un camino no pavimentado con tramos estabilizados con cloruro de calcio y Bischofita. El propósito de estas evaluaciones fue determinar el comportamiento funcional en terreno de capas de rodadura estabilizadas con estos agentes estabilizantes, bajo las mismas condiciones climáticas y de tráfico vehicular.

El camino de prueba estabilizado forma parte de los desvíos de tránsito del proyecto Servicio de mejoramiento de accesibilidad e integración de las villas Tucnar Huasi - Huaytiquina, Calama, que realiza la Corporación Nacional del Cobre de Chile CODELCO, por medio de la empresa contratista Ingeniería Maquinaria y Construcción Ltda. Este desvío proveyó un camino auxiliar mientras 
se realizaban las obras de repavimentación de las vías involucradas (intersección Rutas 24 y 25). Los tramos de prueba corresponden a capas de rodadura estabilizadas con cloruro de calcio y Bischofita. Se encuentran bajo un clima desértico por sobre los $2300 \mathrm{msnm}$, con una humedad relativa que no sobrepasa el 30\% durante el día, mientras que en el transcurso de la noche llega a niveles más altos (55\%).

La extensión del tramo estabilizado con $\mathrm{CaCl}_{2}$ (dosis $1.5 \%$ en peso) es de $1300 \mathrm{~m}$ (Figura 3), mientras que el tramo estabilizado con Bischofita (dosis $6 \%$ en peso), tiene una extensión de $200 \mathrm{~m}$. Ambos tramos poseen un ancho de calzada de $6 \mathrm{~m}$, con espesor de capa de base de $20 \mathrm{~cm}$ (Figura 4) conformada por un suelo tipo A-1-b y pendientes que no sobrepasan el 3\%. Presentan un volumen de tránsito cercano a los $500 \mathrm{veh} /$ día, de los cuales el $40 \%$ corresponde a vehículos pesados de más de 2 ejes, y el resto a vehículos livianos (autos, furgones, jeeps y camionetas).

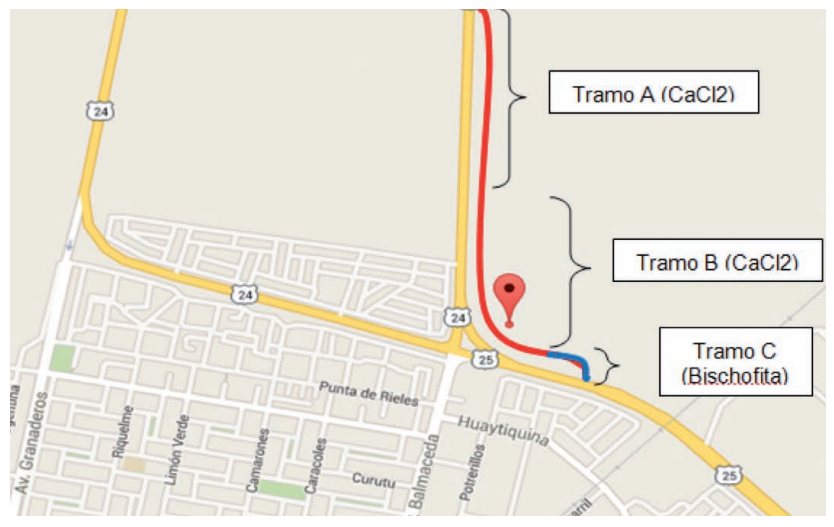

Figura 3: Trazado del tramo a estabilizar con cloruro de calcio y Bischofita

\section{Tránsito Pesado - Clima Seco}

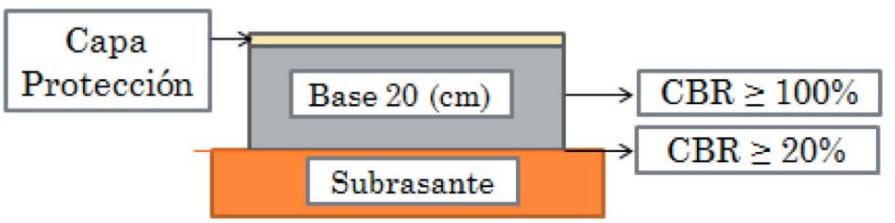

Figura 4: Estructuración del camino estabilizado con cloruro de calcio y Bischofita, Cartilla N 4 (Dirección Nacional de Vialidad, 2002)

\section{Presentación y análisis de resultados}

Para el ensaye Proctor modificado, las Figuras 5a y 5 b muestran que la incorporación de $\mathrm{CaCl}_{2}$ en el suelo aumenta los valores de densidad máxima seca compactada y disminuye los valores de su humedad óptima, respectivamente. En particular con la adición de un 1.5\% de $\mathrm{CaCl}_{2}$ se obtuvieron los mejores resultados, encontrando el mayor aumento de su densidad máxima compactada seca $\left(2.33 \mathrm{~g} / \mathrm{cm}^{3}\right)$, y el menor valor de su humedad óptima $(5.2 \%)$.
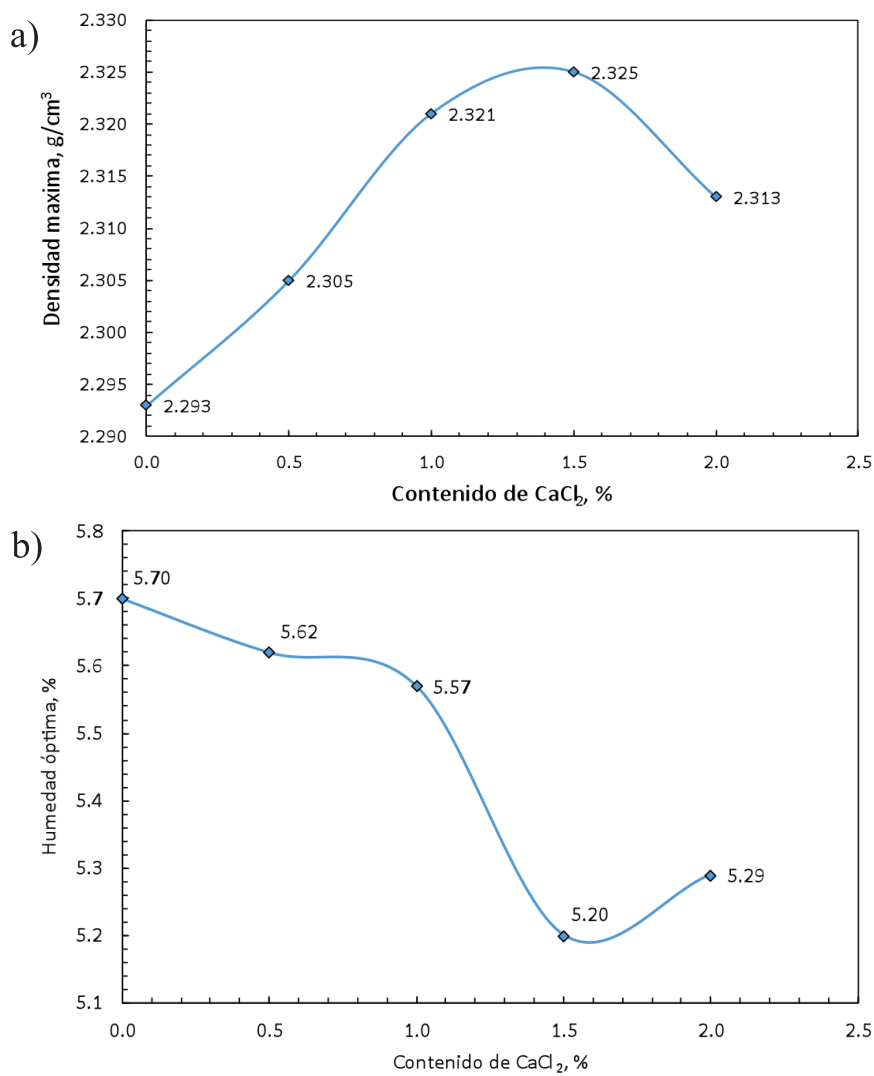

Figura 5: Resultados obtenidos en ensaye Proctor modificado con adición de $\mathrm{CaCl}_{2}$ en el suelo, a) densidad máxima compactada seca y b) humedad óptima

Para el ensaye triaxial CU, las Figuras 6a y $6 \mathrm{~b}$ muestran que la incorporación de $\mathrm{CaCl}_{2}$ en el suelo aumenta los valores del ángulo de fricción interna máximo $\phi$ ' y cohesión $c$, respectivamente. Estos valores aumentan en mayor medida cuando la muestra de suelo con $\mathrm{CaCl}_{2}$ se cura durante 5 días. Para ambos parámetros la adición de un $2 \%$ de $\mathrm{CaCl}_{2}$ con 5 días de curado genera los mejores resultados: $\phi^{\prime}=40.7^{\circ}$ y $c=50 \mathrm{kPa}$.

Para el ensaye CBR medido a $2.54 \mathrm{~mm}$ (0.1") de penetración al 95\% de DMCS, la Figura 7 muestra que la incorporación de $\mathrm{CaCl}_{2}$ en el suelo aumenta los valores de razón de soporte de California CBR. En particular con la adición de un $1.0 \%$ de $\mathrm{CaCl}_{2}$ se obtuvieron los mejores resultados, encontrando el mayor aumento de la razón de soporte CBR de 109 a $151 \%$. 

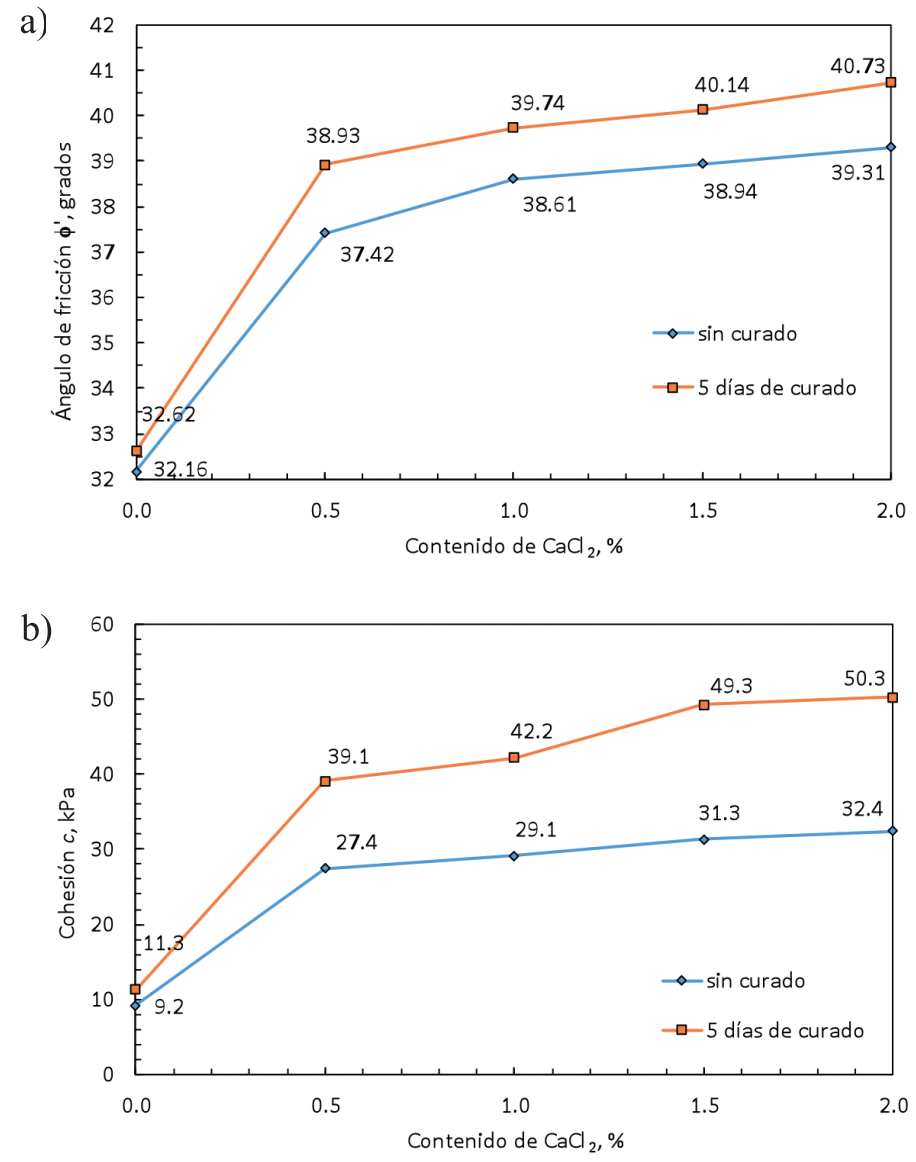

Figura 6: Resultados obtenidos en ensaye triaxial CU con adición de $\mathrm{CaCl}_{2}$ en el suelo, a) ángulo de fricción interna máximo y b) cohesión

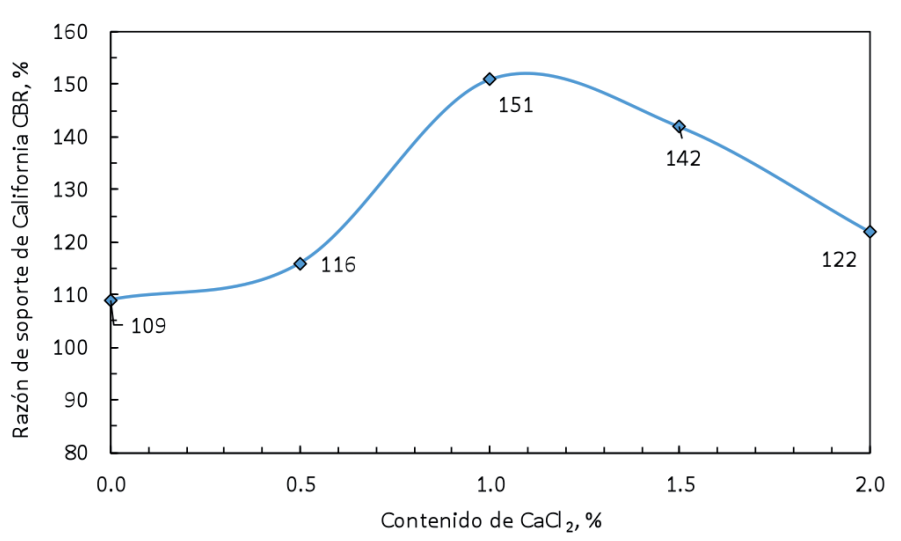

Figura 7: Resultados obtenidos de ensaye CBR con adición de $\mathrm{CaCl}_{2}$ en el suelo

Para el ensaye de absorción de humedad, la Figura 8 muestra que en la medida que se incorpora $\mathrm{CaCl}_{2}$ en el suelo, aumenta la cantidad de agua absorbida. Con la adición de un $2.0 \%$ de $\mathrm{CaCl}_{2}$ se obtuvieron los mejores resultados, encontrando la mayor cantidad de agua en el suelo (22.2 $\mathrm{g}$ de agua absorbida, en una muestra de $6000 \mathrm{~g}$ compactada al $95 \%$ de la DMSC).

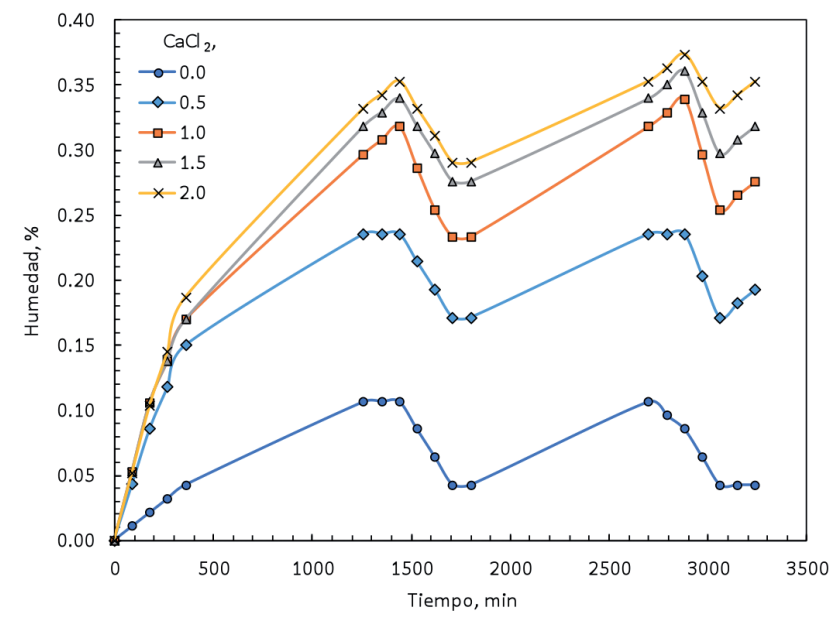

Figura 8: Resultados obtenidos de ensaye de absorción humedad con adición de $\mathrm{CaCl}_{2}$ en el suelo

\section{Evaluación de tramos de prueba}

Para evaluar el comportamiento funcional del camino estabilizado en un tramo con cloruro de calcio y en otro con Bischofita, se realizó un seguimiento periódico con el propósito de determinar de forma cualitativa y cuantitativa el estado del camino. Además, se realizaron pruebas para medir la emanación de polvo desde la superficie del camino, determinando así la eficiencia de la estabilización con cloruro de calcio.

\section{Estado de deterioro del camino}

Para determinar el estado de deterioro del camino de prueba durante el periodo de seguimiento, se utilizó un método presentado por la Dirección Nacional de Vialidad (2011), en el cual se determina el Índice de Condición de caminos No Pavimentados (ICNP) para caminos de tierra a partir del tipo, cantidad, y severidad de las fallas registradas en su superficie (Figura 9).

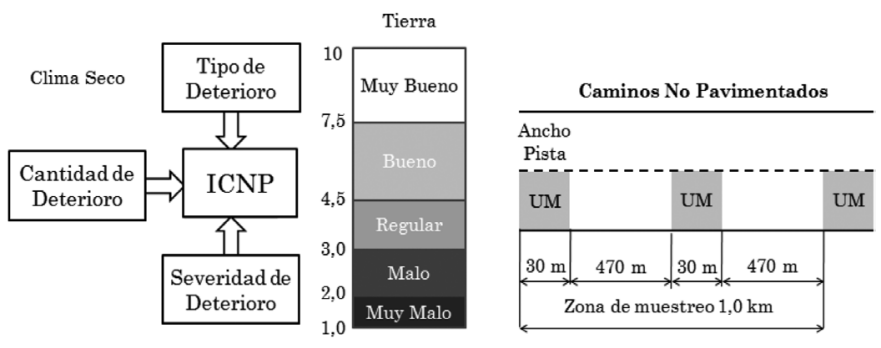

Figura 9: Metodología, escala de calificación y unidad de muestreo para la determinación de estado de deterioro del camino estabilizado con cloruro de calcio y Bischofita (Dirección Nacional de Vialidad, 2011) 
Esta metodología sugiere tomar la Unidad de Muestreo UM como un área rectangular de ancho igual al ancho de la pista y $30 \mathrm{~m}$ de largo. En cada kilómetro inspeccionado es necesario medir 2 Unidades de Muestreo UM, de modo de completar una muestra de $60 \mathrm{~m}$ de largo por kilómetro.

La ecuación para representar el ICNP, sin equipos de auscultación se muestra a continuación:

$\mathrm{ICNP}=10-1.15(1.01$ Calamina +1.96 Baches

+1.28 Erosión +0.29 Ahuellamiento +

1.36 Camino Pedregoso +1.37 Bombeo)

donde en Calamina se ingresa la profundidad media en cm; Baches $=$ profundidad media en $\mathrm{m}$ del bache $\mathrm{x}$ diámetro medio en $\mathrm{m} \times \mathrm{N}^{\circ}$ de baches en UM Erosión se ingresa 1 si la erosión es importante y 0 si no lo es; Ahuellamiento se ingresa la profundidad media en cm; Bombeo (drenaje) se cuantifica con 1 si el bombeo es bueno (perfil transversal cóncavo), con 0.5 si es regular y con 0 si es malo (perfil transversal convexo y/o con irregularidades); Camino Pedregoso se ingresa 1 si el deterioro se detecta y 0 si no. De acuerdo a lo registrado en terreno se tienen los datos mostrados en la Tabla 1.

Tabla 1: Características de las fallas en las unidades de muestreo UM del camino de prueba para índice de condición de camino no pavimentado ICNP

\begin{tabular}{|c|c|c|c|c|c|}
\hline & \multirow{2}{*}{\multicolumn{4}{|c|}{ Unidad de Muestreo UM }} \\
\hline & & & & & \\
\hline & & 1 & 2 & 3 & 4 \\
\hline \multicolumn{2}{|c|}{$\begin{array}{l}\text { Tipo de } \\
\text { estabilización }\end{array}$} & Bischofita & $\mathrm{CaCl}_{2}$ & $\mathrm{CaCl}_{2}$ & $\mathrm{CaCl}_{2}$ \\
\hline \multicolumn{2}{|c|}{ Distancia, km } & $0.0-0.2$ & $0.2-0.7$ & $0.7-1.2$ & $1.2-1.5$ \\
\hline \multicolumn{2}{|c|}{ Drenaje (bombeo) } & $\begin{array}{c}\text { Perfil } \\
\text { transversal } \\
\text { semicón- } \\
\text { cavo } \\
\end{array}$ & \begin{tabular}{|c|} 
Perfil \\
transversal \\
semicón- \\
cavo \\
\end{tabular} & $\begin{array}{c}\text { Perfil } \\
\text { transversal } \\
\text { semicón- } \\
\text { cavo } \\
\end{array}$ & \begin{tabular}{|c} 
Perfil \\
transversal \\
semicón- \\
cavo \\
\end{tabular} \\
\hline \multicolumn{2}{|c|}{ Calamina } & $\begin{array}{c}\text { No } \\
\text { presenta } \\
\end{array}$ & $\begin{array}{c}\text { No } \\
\text { presenta }\end{array}$ & $\begin{array}{c}\text { No } \\
\text { presenta } \\
\end{array}$ & $\begin{array}{c}\text { No } \\
\text { presenta }\end{array}$ \\
\hline \multicolumn{2}{|c|}{ Ahuellamiento, $\mathrm{cm}$} & 0.6 & 0.5 & 0.5 & 0.6 \\
\hline \multicolumn{2}{|c|}{ Camino pedregoso } & $\begin{array}{l}\text { Presenta } \\
\text { árido } \\
\text { grueso } \\
\text { suelto }\end{array}$ & $\begin{array}{c}\text { No } \\
\text { presenta } \\
\text { árido } \\
\text { grueso } \\
\text { suelto }\end{array}$ & $\begin{array}{c}\text { No } \\
\text { presenta } \\
\text { árido } \\
\text { grueso } \\
\text { suelto }\end{array}$ & $\begin{array}{l}\text { Presenta } \\
\text { árido } \\
\text { grueso } \\
\text { suelto }\end{array}$ \\
\hline \multirow{3}{*}{ Baches } & $\mathrm{N}^{\circ}$ baches & 30 & 10 & 10 & 5 \\
\hline & $\begin{array}{l}\text { Profundi- } \\
\text { dad media } \\
\text { baches, cm }\end{array}$ & 5 & 3 & 3 & 5 \\
\hline & $\begin{array}{l}\text { Diámetro } \\
\text { medio } \\
\text { baches, } \mathrm{cm} \\
\end{array}$ & 10 & 5 & 6 & 6 \\
\hline \multicolumn{2}{|l|}{ Erosión } & $\begin{array}{c}\text { Erosión } \\
\text { baja }\end{array}$ & $\begin{array}{c}\text { Erosión } \\
\text { baja }\end{array}$ & $\begin{array}{c}\text { Erosión } \\
\text { baja }\end{array}$ & $\begin{array}{c}\text { Erosión } \\
\text { baja }\end{array}$ \\
\hline
\end{tabular}

De acuerdo a los datos de entrada de la Tabla 1, el índice de condición de camino no pavimentado ICNP para los tramos estabilizados con cloruro de calcio y Bischofita se presenta en las Tablas 2 y 3.

De acuerdo a los resultados obtenidos en la evaluación del estado de deterioro del camino, se tiene que los tramos estabilizados con $\mathrm{CaCl}_{2}$ obtuvieron mejor calificación que el tramo estabilizado con Bischofita, obteniendo como promedio nota 8 y la Bischofita nota 5.6. En este sentido el desempeño del cloruro de calcio como agente estabilizador fue mejor que la Bischofita. Se observa que las estabilizaciones con $\mathrm{CaCl}_{2}$ y Bischofita permiten eliminar la liberación de polvo, corrugaciones y textura gruesa, y reducir la formación de baches y pérdida de material.

Tabla 2: Planilla de determinación del estado de caminos no pavimentados (1)

\begin{tabular}{|c|c|c|c|c|c|c|c|c|c|}
\hline \multicolumn{7}{|c|}{ Evaluación camino tierra estabilizado con $\mathrm{CaCl}_{2}$ - día 100} \\
\hline $\mathrm{UM}$ & $\begin{array}{c}\text { Tipo de } \\
\text { estabiliza- } \\
\text { ción }\end{array}$ & $\begin{array}{c}\mathrm{km} \\
\text { (i) }\end{array}$ & $\begin{array}{c}\mathrm{km} \\
\text { (f) }\end{array}$ & $\begin{array}{c}\text { Drenaje } \\
\text { (bombeo }) \\
(00.5 \text { ó } 1)\end{array}$ & $\begin{array}{c}\text { Calami- } \\
\text { nas, cm }\end{array}$ & $\begin{array}{c}\text { Ahuell.. } \\
\mathrm{cm}\end{array}$ & $\begin{array}{c}\text { Camino } \\
\text { pedregoso } \\
\text { (0 ó 1) }\end{array}$ & $\begin{array}{c}\text { Baches, } \\
\mathrm{m}^{2}\end{array}$ & $\begin{array}{c}\text { Erosión } \\
(0 \text { ó 1) }\end{array}$ \\
\hline 1 & Bischofita & 0.0 & 0.2 & 0.5 & 0 & 0.6 & 1 & 0.150 & 1 \\
\hline 2 & $\mathrm{CaCl}_{2}$ & 0.2 & 0.7 & 0.5 & 0 & 0.5 & 0 & 0.015 & 1 \\
\hline 3 & $\mathrm{CaCl}_{2}$ & 0.7 & 1.2 & 0.5 & 0 & 0.5 & 0 & 0.018 & 0 \\
\hline 4 & $\mathrm{CaCl}_{2}$ & 1.2 & 1.5 & 0.5 & 0 & 0.6 & 1 & 0.015 & 0 \\
\hline
\end{tabular}

Tabla 3: Planilla de determinación del estado de caminos no pavimentados (2)

\begin{tabular}{|c|c|c|c|c|}
\hline & \multirow{2}{*}{$\begin{array}{c}\text { Calificación } \\
\text { cuantitativa }\end{array}$} & \multicolumn{3}{|c|}{$\begin{array}{c}\text { Calificación cualitativa del estado por zona } \\
\text { climática }\end{array}$} \\
\hline \multirow{2}{*}{ UM } & \multirow{2}{*}{$\begin{array}{c}\text { Nota UM } \\
\text { (1 a 10) }\end{array}$} & Seca & Mediterránea & Húmeda \\
\cline { 3 - 5 } & 5.6 & Bueno & Bueno & Regular \\
\hline 1 & 7.5 & Muy bueno & Bueno & Bueno \\
\hline 2 & 9.0 & Muy bueno & Muy bueno & Muy bueno \\
\hline 3 & 7.4 & Bueno & Bueno & Bueno \\
\hline 4 & & & &
\end{tabular}

En ambos tramos se produjeron agrietamiento de la superficie de rodadura del tramo de prueba como se puede observar en la Figura 10. La causa probable del agrietamiento es la retracción del material granular debido al incremento de la tensión superficial junto a una baja humedad relativa prevaleciente en la zona. Aunque no existe la humedad suficiente para que la superficie de rodadura la absorba, no hay liberación de polvo, baches ni calaminas, esto es consecuencia del mecanismo de 
cementación de partículas finas en la superficie de rodadura (por cristalización de las sales). Los tramos de prueba presentan una excelente calidad de rodadura que permite circular a una velocidad de $60 \mathrm{~km} / \mathrm{h}$.
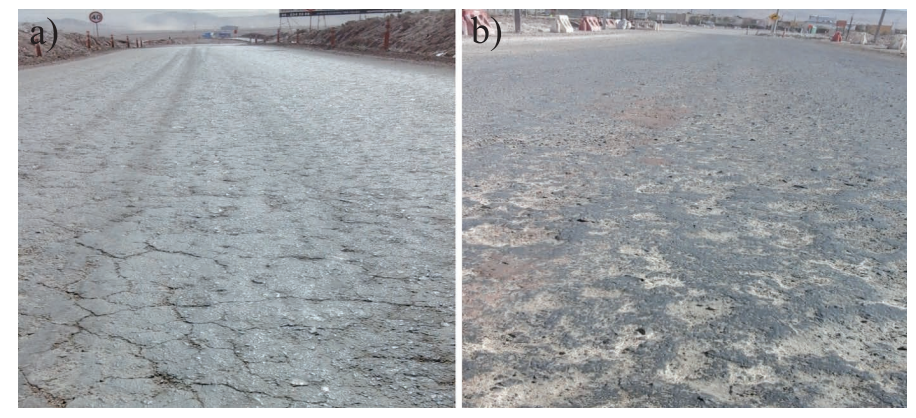

Figura 10: Fallas en el tramo estabilizado con: a) $\mathrm{CaCl}_{2}$ y b) Bischofita

\section{Mitigación de polvo}

Con el objeto de cuantificar la eficiencia de la aplicación de $\mathrm{CaCl}_{2}$ en la supresión de material particulado PM10 producido por el paso de los vehículos, se utilizó la metodología de Medición de Polvo en Camino MPC. El parámetro $\mathrm{PM}(\mathrm{x})$ define la cantidad de partículas de tamaño menor a $(\mathrm{x})$ micrones que posee un centímetro cúbico de aire.

El levantamiento se lleva a cabo con un procedimiento que utiliza el equipo Dustmate mostrado en la Figura 11a, proporcionado por la empresa patrocinadora de la presente investigación, Ventech Ltda. El equipo de medición de polvo está especialmente diseñado para cuantificar la concentración de partículas de tamaño menor a PM1, PM2.5 y PM10. Las partículas que posee la muestra de aire son analizadas por un fotómetro láser, el cual permite cuantificar la cantidad de partículas según el tamaño especificado. La muestra de aire se toma detrás de la rueda trasera del vehículo, de manera de obtener directamente la cantidad de polvo generada por la tracción del neumático sobre la superficie del camino como se muestra en la Figura $11 \mathrm{~b}$.

El 3 de febrero del año 2016, se realizaron mediciones en el tramo no estabilizado, previo a la construcción de la carpeta estabilizada. Estas mediciones de PM10 corresponden al número de partículas de tamaño menor a 10 micrones por centímetro cúbico. A partir del análisis de la información, se establece como línea base un valor PM10 de 4287 pcc.

En el gráfico de la Figura 12, se muestran las emisiones promedio semanal del tramo estabilizado con $\mathrm{CaCl}_{2}$ a partir del día 16 de marzo de 2016 (inicio de mediciones en tramo estabilizado) hasta el día 8 de junio de 2016. Además, se aprecia la línea base de emisión (en color rojo), que demuestra las diferencias de un camino con adición de cloruro de calcio y sin la aplicación de un supresor de polvo. Durante un periodo de 3 meses se realizaron mediciones semanales obteniendo los valores y eficiencias mostrados en la Figura 12. Los resultados obtenidos de la eficiencia en la mitigación de polvo en el periodo fueron:

- Eficiencia promedio: $85.3 \%$ (respecto al estado inicial)

- Eficiencia máxima: 97.6\% (respecto al estado inicial)

- Eficiencia mínima: 65.2\% (respecto al estado inicial)

a)
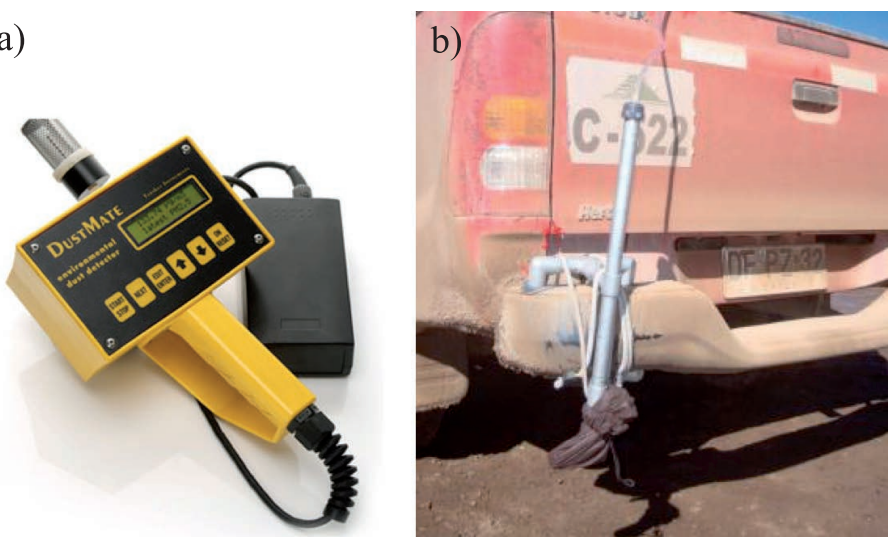

Figura 11: a) Equipo Dustmate y b) medición de partículas detrás de un vehículo

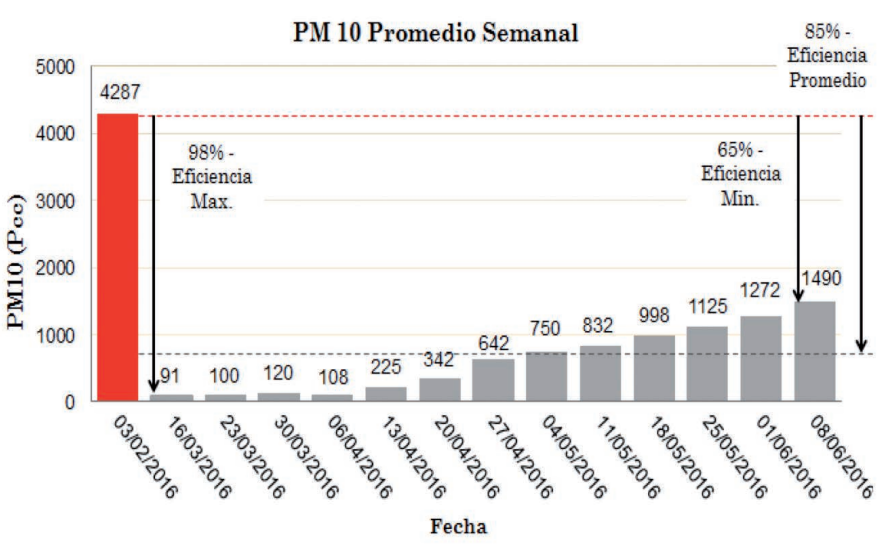

Figura 12: Mediciones de polvo PM10 promedio semanal en el camino estabilizado con $\mathrm{CaCl}_{2}$

En la Figura 13 se aprecia el comportamiento de la carpeta de rodado ante el paso de un vehículo pesado que normalmente genera levantamiento de polvo en caminos no pavimentados. 

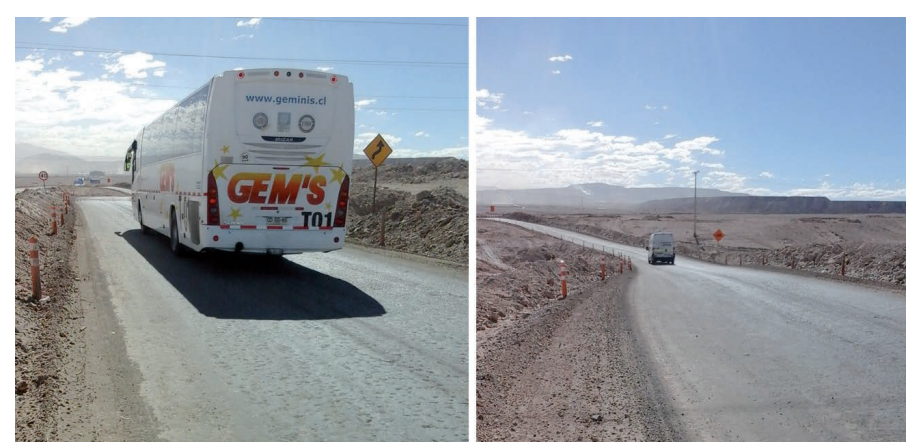

Figura 13: Emanación de polvo de la superficie del camino, semana 20 abril de 2016, PM10 = 342 Pcc

\section{Evaluación económica}

Para determinar el costo de implementación de la estabilización con cloruro de calcio y Bischofita, se realizó un análisis de precios unitarios. Se debe considerar que el proceso constructivo de ambas estabilizaciones es similar, difiriendo únicamente en el costo de aplicación y costo de mantención. De acuerdo a esta situación, se obtuvieron los costos en pesos chilenos por $\mathrm{m}^{2}$ de aplicación y mantención de base estabilizada con ambas sales, lo cual se resume en la Tabla 4.

Tabla 4: Costos por $\mathrm{m}^{2}$ de aplicación y mantención de estabilización con $\mathrm{CaCl}_{2}$ y Bischofita (abril de 2016)

\begin{tabular}{|c|c|c|c|}
\hline $\begin{array}{c}\text { Actividad/ } \\
\text { Estabilizante }\end{array}$ & $\begin{array}{c}\text { Costo de } \\
\text { aplicación por } \\
\mathrm{m}^{2} \text { de base } \\
\text { granular, } \$\end{array}$ & $\begin{array}{c}\text { Costo de } \\
\text { mantención } \\
\left(\mathrm{m}^{2}\right) \text { carpeta, } \$\end{array}$ & Observación \\
\hline $\mathrm{CaCl}_{2}$ & 952 & 460 & Cada 10 meses \\
\hline Bischofita & 815 & 350 & Cada 8 meses \\
\hline
\end{tabular}

Para determinar el costo proyectado en el tiempo de las estabilizaciones con $\mathrm{CaCl}_{2}$ y Bischofita, se debe tener presente la frecuencia de mantenciones que se deben realizar a cada caso bajo las condiciones climáticas y de tráfico del presente estudio. Para esto se ha proyectado, de acuerdo a lo observado en terreno, que la estabilización con $\mathrm{CaCl}_{2}$ tiene una frecuencia de mantención de 10 meses, con una reparación total cada 4 años. Bajo estas consideraciones se aprecia en el gráfico de la Figura 14 una proyección para un periodo de 7 años.

De acuerdo al estudio económico para un camino de $1 \mathrm{~km}$ con pista de ancho $6 \mathrm{~m}$, se demuestra que al término de cada año (hasta el tercer año) la estabilización con Bischofita presenta mejores resultados en el costo acumulado que la

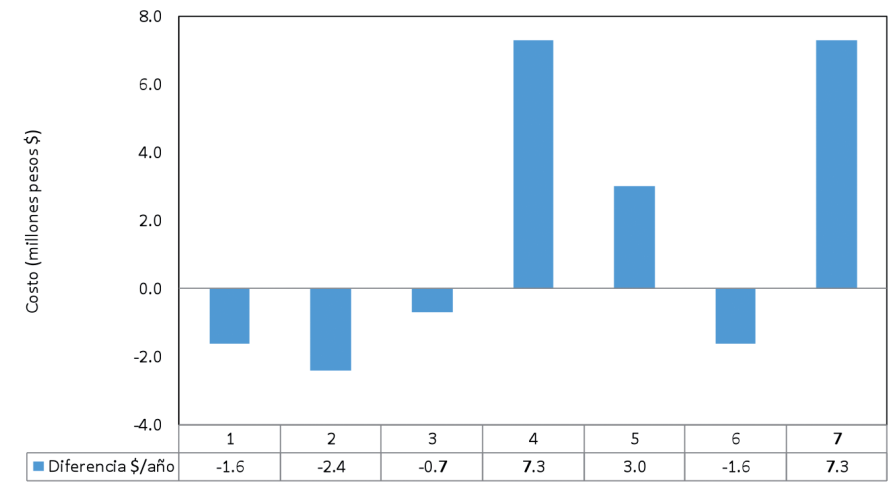

Figura 14: Proyección de costos de estabilización con $\mathrm{CaCl}_{2} \mathrm{y}$ Bischofita por cada año (millones de pesos chilenos)

estabilización con $\mathrm{CaCl}_{2}$, llegando a un ahorro de un $6.5 \%$ al cabo del primer año, un $8.6 \%$ al cabo del segundo año y un $2.2 \%$ al cabo del tercer año. Esta situación cambia al cuarto año de estabilizado el camino, puesto que los costos de estabilizar con $\mathrm{CaCl}_{2}$ resultan más convenientes que los obtenidos con la utilización de Bischofita. El costo acumulado de la estabilización con Bischofita hasta dicho periodo asciende a un valor de $\$ 43.8$ millones $/ \mathrm{km}$, mientras que el valor de la estabilización con cloruro de calcio asciende a $\$ 36.5$ millones $/ \mathrm{km}$, evidenciando un ahorro favorable al $\mathrm{CaCl}_{2}$ de un $20 \%$, situación que se mantiene hasta el término del quinto año con un ahorro en el costo acumulado de $\$ 3.0$ millones (6.5\%) favorable a la estabilización con $\mathrm{CaCl}_{2}$.

Al sexto año la situación cambia, puesto que el costo acumulado favorece la estabilización con Bischofita con un ahorro de $\$ 1.6$ millones (3\%), cambiando abruptamente al término del séptimo año, presentándose un ahorro de 7.3 millones (12.5\%) favorable a la estabilización con $\mathrm{CaCl}_{2}$.

Debe considerarse que la evaluación económica depende también de la frecuencia de mantenimiento, las que varían según el tipo de camino y condiciones climáticas.

\section{Conclusiones}

- Las mediciones en terreno muestran que el cloruro de calcio es un efectivo estabilizador de capas granulares en climas áridos, reduciendo la liberación de polvo, retardando la formación de calamina y baches, y reduciendo la pérdida de material, generando una excelente calidad de rodadura cercana a la de caminos pavimentados. 
- La calidad final de la superficie depende de la construcción, en particular, de la adecuada homogeneización del suelo con el producto y de la terminación superficial de la capa de rodadura.

- El estado del camino según el Índice de Condición de Caminos No Pavimentados ICNP a los 100 días, es Muy bueno $\rightarrow$ ICNP promedio de 8 para el tramo estabilizado con $\mathrm{CaCl}_{2}$, y Bueno $\rightarrow$ ICNP de 5.6 para el tramo con Bischofita. En este sentido el tramo estabilizado con $\mathrm{CaCl}_{2}$, obtuvo una calificación cualitativa mayor, bajo las mismas condiciones de tránsito y ambientales.

- Bajo condiciones de clima árido, baja humedad relativa, y un tránsito de $500 \mathrm{veh} /$ día (30\% tránsito pesado), el camino estabilizado con $\mathrm{CaCl}_{2}$ proyecta una duración sin mantenciones de 10 a 11 meses, con reparaciones completas cada 4 años, mientras que el camino estabilizado con Bischofita proyecta una duración sin mantenciones de 8 a 9 meses, con reparaciones completas cada 3 años.

- La capacidad de soporte del suelo mejora con la adición de cloruro de calcio. Estos valores se incrementaron a medida que el proceso de curado del suelo se fue completando, en mayor medida con la adición de un $1 \%$ de esta sal.

- El aumento de humedad en el suelo está relacionado con la adición de cloruro de calcio. El contenido de humedad aumenta en la medida que aumenta la cantidad de $\mathrm{CaCl}_{2}$, sin embargo, una adición mayor al $1.5 \%$ no es conveniente desde el punto de vista económico.

- La estabilización con cloruro de calcio fue eficiente en la mitigación de polvo generado por los vehículos, llegando a una media del $85 \%$. Durante las últimas semanas de medición, la eficiencia fue descendiendo llegando al $65 \%$, debido a que los índices de humedad relativa durante ese último periodo fueron descendiendo.

- La estabilización con cloruro de calcio genera mejoras en las propiedades mecánicas del suelo. Una dosificación entre $1 \%$ y $1.5 \%$ entrega al suelo los mejores beneficios económicos y en sus propiedades resistentes.

- El estudio económico reveló que a corto plazo (2 años), bajo condiciones climáticas adversas y alto tránsito vehicular, la estabilización con Bischofita resultaría un
8.6\% más económica que la estabilización con cloruro de calcio.

- A mediano plazo (7 años) bajo condiciones climáticas adversas y alto tránsito vehicular, la estabilización con cloruro de calcio resultaría un $20 \%$ más económica que estabilizar con Bischofita.

- Se puede aprovechar el suelo existente reduciendo la necesidad de transporte y material de empréstitos, lo que deviene en una componente ambiental positiva. No se tiene antecedentes de que el cloruro de calcio genere contaminación ambiental dada las bajas concentraciones utilizadas en el camino. Con respecto a la Bischofita ampliamente utilizada en la zona Norte, no hay antecedentes de posibles efectos contaminantes.

\section{Referencias}

Botasso, G. y Segura, A. (2013). Estudio experimental de microaglomerado asfáltico antiderrapante modificado con NFU. Obras y Proyectos 14, 36-44

Cortés, E. (2002). Aplicación en terreno y evaluación de estabilizadores químicos de suelo en ruta no pavimentada de la provincia del Loa, II Región Antofagasta. Memoria de título de Ingeniero Civil, Universidad Católica del Norte, Antofagasta, Chile

Crespo, C. (1998). Mecánica de suelos y cimentaciones. Limusa, México

Dirección Nacional de Vialidad (2011). Política de conservación vial. Etapa 3 - caminos no pavimentados. Ministerio de Obras Públicas, Gobierno de Chile

Dirección Nacional de Vialidad (2002). Guía de diseño estructural de pavimentos para caminos de bajo volumen de tránsito. Informe desarrollado por la PUC para el MOP, Chile

Fernández, C. (1982). Mejoramiento y estabilización de suelos. Limusa, México

Mateos, M. (2007). Efectos del cloruro cálcico en la estabilidad de las tierras. Cimbra 373, 44-48

Monlux, S. (2003). Stabilizing unpaved roads with calcium chloride. Transportation Research Record 1819(1), 52-56

MOP (2014). Informe Red Vial Nacional año 2014-2015. Dirección de Vialidad, Chile 
Occidental Chemical Chile Ltda. (2012). Manual cloruro de calcio $\mathrm{CaCl}_{2}$. Providencia, Chile

Orobio, A., Portocarrero, L.M.P. y Serna, L. (2007). Evaluación del cloruro de calcio como agente mitigador de polvo en vías en afirmado. DYNA 74(153), 27-33

Quinche, W.O. (2006). Estabilización de suelos con cloruro de sodio para su uso en vías terrestres urbanización Bella María. Tesis doctoral, Universidad Técnica Particular de Loja, Ecuador
Thenoux, G. y Vera, S. (2002). Evaluación de la efectividad del cloruro de magnesio hexahidratado (Bischofita) como estabilizador químico de capas de rodadura granulares. Materiales de Construcción 52(265), 5-22 